\title{
An Audit Logic for Accountability (Extended Version*)
}

\author{
J.G. Cederquist ${ }^{1}$, R. Corin ${ }^{1}$, M.A.C. Dekker ${ }^{1,2}$, S. Etalle ${ }^{1}$ and J.I. den Hartog ${ }^{1}$ \\ ${ }^{1}$ Department of Computer Science, University of Twente, The Netherlands \\ \{cederquistj, corin, etalle, hartogji\}@cs.utwente.nl \\ ${ }^{2}$ Security Department, TNO ICT, The Netherlands \\ m.a.c.dekkeretelecom.tno.nl
}

\begin{abstract}
We describe a policy language and implement its associated proof checking system. In our system, agents can distribute data along with usage policies in a decentralized architecture. Our language supports the specification of conditions and obligations, and also the possibility to refine policies. In our framework, the compliance with usage policies is not actively enforced. However, agents are accountable for their actions, and may be audited by an authority requiring justifications.
\end{abstract}

\section{Introduction}

In many situations, there is a need to share data between potentially untrusted parties while ensuring the data is used according to given policies. This problem is addressed by two main research streams: on one hand, there is a large body of literature on access (and usage) control [8, 16, 11, 4], on the other hand we find digital rights management [18, 5]. While the former assumes a trusted access control service restricting data access, the latter assume trusted devices in charge of content rendering. Both settings need the trusted components to be available at the moment the request happens, to regulate the data access.

However, there are scenarios (like the protection of private data) in which both access control and digital rights management fail, either because the necessary trusted components are not available or because they are controlled by agents we do not want to trust. For instance, P3P [17] and E-P3P (and also EPAL) [3] are languages that allow one to specify policies for privacy protection; however, the user can only hope that the private data host follows them.

In this paper, the process of regulating the data access is not assumed to be always performed by the same entity at the same moment in which the access is requested. More specifically, we relax this in mainly two ways:

- Firstly, at the moment that the data is requested, we assume that access is always granted, and only later it is determined whether the requestor had permission to access the data. This is the process of auditing. To achieve this, we need all the relevant decision information to be kept until audit time (e.g. keeping secure logs).

- Secondly, the entity that is performing the auditing does not need to be fixed, and can thus be dynamically chosen. This is useful since, for example, some authorities are more appropiate to audit specific agents than others. The actual authority does not even need to be one single entity, and can be for example composed of regular agents.

We present a flexible system which allows to express, deploy and reason about policies controlling the usage of data. In our target setting agents can distribute data along with usage policies within a highly decentralized architecture, in which the enforcement of policies is difficult (if not impossible). Therefore, we use instead an auditing system with best-effort checking by an authority which is able to observe (some) actions. We introduce a notion of agent accountability and express the proof obligation of an agent being audited. The system allows to reason about policies and user accountability. Our framework is depicted in Figure 1

\footnotetext{
${ }^{*}$ This is an extended version of the article to appear in the Conference Proceedings of the 6th IEEE International Workshop on Policies for Distributed Systems and Networks (POLICY 2005)
} 


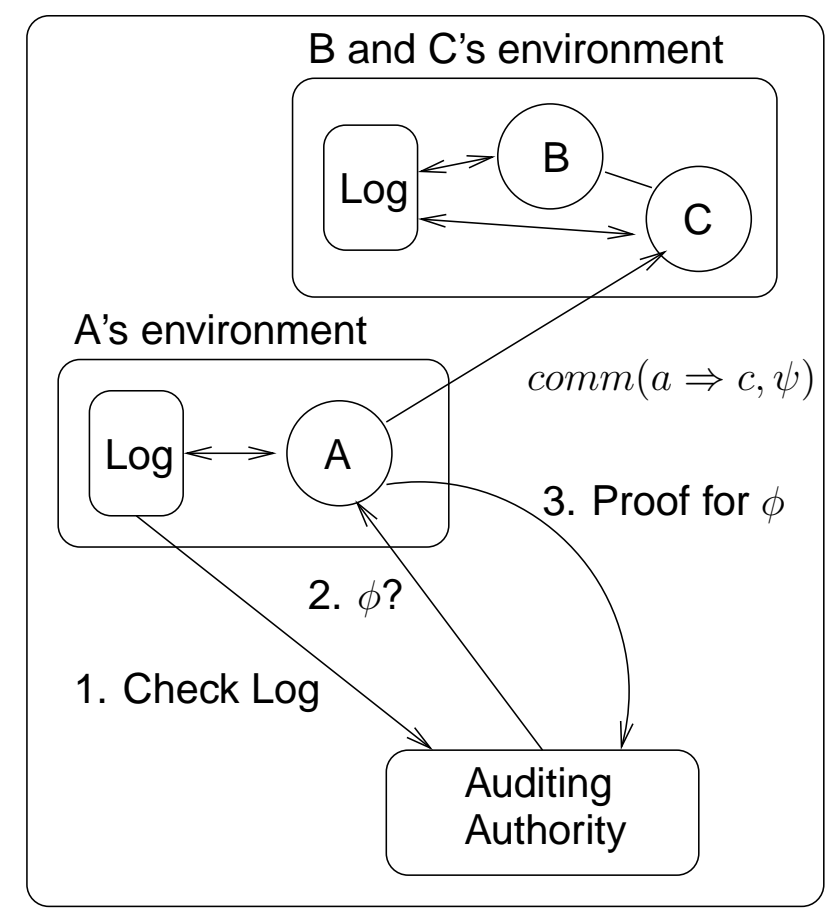

Figure 1. Our framework

We make no assumptions on the existence of trusted components regulating access (although we do require a trusted environment to certify environmental conditions, and to securely log events). In fact, agents are not forced to follow the policies, but may be audited by authorities which ask for justifications. We make no particular assumptions about authorities; they may comprise, for instance, of groups of regular agents. The more an authority can observe, the more accurate the auditing process is, thus providing more confidence over the agent's behaviour. To characterize compliant agent behaviour, as perceived by an authority, we define accountability tests, which are carried out during auditing by the authority.

Of course, our approach does not allow a strict policy enforcement: agents can easily "misbehave" (i.e. treat data in a way that is not allowed by the policy), at risk of being traced. It is our belief that in many emerging scenarios active policy enforcement is infeasible.

This paper builds on the preliminary work reported in [6]. In particular, we provide several extensions, the most notable of which are:

- We include the ability to specify conditions and obligations within the policies.

- Policies may now contain variables and quantifiers. This allows us to define a fundamental rule that gives the ability to refine policies. Agents can create (by refinement) new policies from existing ones, before passing them to other agents. In contrast, in [6] the only policies allowed are those that are explicitly stated by the data owner.

- We precisely describe our system by introducing three functions, namely the observability, conclusion derivation and proof obligation functions. Moreover, we provide a customizable action set to account for particular, user-defined scenarios.

- We define agent accountability tests, and present a (terminating) procedure for recursive auditing.

- Finally, we provide a formalization of our proof system in the proof checker Twelf[13], which allows us to model proofs provided by agents, and the subsequent checking by the authority (Our formalization covers the lower part of Figure1) 


\section{A System of Policies and Actions}

Our setup consists of a group of agents executing different actions. The permission to execute an action is expressed by a policy constructed using a special logic, introduced below. In this section we introduce some necessary components for our system.

\subsection{The basics}

Agents are modelled by a set $\mathcal{G}$ ranged over by $a, b$ and $c$ (referred to as Alice, Bob, and Charlie). We also have a set of agent variables $\mathcal{V}_{a}$ and use $A, B, C$ to range over both agents and agent variables. Similarly we have a set $\mathcal{D}$ of data objects, ranged over by $d$, and a set of data variables $\mathcal{V}_{d}$. We use $D$ to range over data objects and data variables and $x, y, z$ to range over (data and agent) variables.

Basic permissions and facts are expressed by atomic predicates in a set $\mathcal{C}$, ranged over by $p$. Examples are read $(a, d)$, which expresses that agent $a$ has permission to read data $d$ and partner $(a, b)$ indicating (the fact) that agent $a$ and $b$ are partners. In general, predicates can relate any number of data objects and agents.

The actions that agents execute are modelled using a set of actions $A C T$, ranged over by act. We assume that two types of actions are always present in this set: Communication (of policies) $\operatorname{comm}(a \Rightarrow b, \phi)$ and data creation creates $(a, d)$. (Here $a, b$ are agents and $\phi$ is a ground policy formula, as introduced in the next subsection). Our system supports the addition of user-defined actions.

\subsection{The Policy Language}

Policies are used to express permissions that agents have, such as the permission to read a specific piece of data. Some requirements may guard a permission. These requirements can be conditions, as in 'Alice may read the data if she is a partner of Bob', or obligations, as in 'Alice may read the data if she pays Bob 10\$'. Besides this, a policy may express or relate several different permissions. To provide maximum flexibility for writing policies, we now introduce the following policy language.

Definition 1 The set of policies $\Phi$, ranged over by $\phi$ and $\psi$, is defined by the following grammar:

$$
\begin{aligned}
\phi::= & p\left(s_{1}, \ldots, s_{n}\right) \\
& \mid A \text { owns } D \mid A \text { says } \phi \text { to } B \\
& |\phi \wedge \phi| \forall x . \phi|\phi \rightarrow \phi| \xi \rightarrow \phi \\
s::= & A \mid D \\
\xi::= & \text { !act } \mid \text { ?act }
\end{aligned}
$$

First, a policy formula can be a simple predicate $p\left(s_{1}, \ldots, s_{n}\right)$, where $s_{i}$ 's can be either an agent, an agent variable, a data object or a data object variable. Second, we have the $A$ owns $D$ formula, which indicates that $A$ is the owner of data object $D$. As we define below, an data owner is allowed to create usage policies related to that data. $A$ says $\phi$ to $B$ expresses that agent $A$ is allowed to give policy $\phi$ to agent $B$. The 'says' policy contains a target agent to which the statement is said (different from e.g. [7 [1]). This allows us to provide a precise way of communicating policies to certain agents. However, the policy $A$ says $\phi$ to $B$ carries a different meaning for source agent $A$ than target agent $B$ : While for agent $A$ it represents the permission to send $\phi$ to $B$, for $B$ it represents the possibility to use policy $\phi$ and delegate the responsibility to $A$.

The logic constructions and, implication and universal quantification have their usual meaning. We actually have two different instances of the implication. The first, $\phi^{\prime} \rightarrow \phi$, has a policy $\phi^{\prime}$ as a condition, stating that the agent first needs to establish this permission or fact before gaining the permission described in $\phi$. The second, $\xi \rightarrow \phi$, is used to express obligations. The requirement $\xi$ contains an action that the agent has to perform when the permission granted by $\phi$ is used. The annotations! and ? indicate whether the agent needs to do this action every time it uses $\phi$ or only once. This will be discussed in Section 3.3 We write $\phi[D]$ to indicate that the set $D$ is the data set of $\phi$, i.e. all data objects and data variables occurring in $\phi$. For instance, we have read $(b, d)[\{d\}]$.

Example 1 The (atomic) policy that allows Bob to read the data $d$ is read $(b, d)$. 
1. The policy that allows Bob to read every data object owned by Alice is $\forall x$. (a owns $x \rightarrow \operatorname{read}(b, x))$.

2. Let age 21(x) denote that agent $x$ is at least 21 years old, and alc $(y)$ denote that beverage $y$ is alcoholic. A policy allowing people over 21 to drink alcoholic beverages is $\forall x, y$. (age $21(x) \wedge \operatorname{alc}(y)) \rightarrow \operatorname{drink}(x, y)$.

3. If we require a payment of $10 \$$ on the previous permission, the policy becomes $\forall x$.(!paid $(x, 10 \$) \rightarrow \forall y$. (age $21(x) \wedge$ $\operatorname{alc}(y)) \rightarrow \operatorname{drink}(x, y))$.

\subsection{Actions and permissions}

To distinguish different instances of an action executed in the system, we label each instance using a unique identifier $i d$, as in creates ${ }_{i d}(a, d)$. This formally gives a set $A C=\mathbb{N} \rightarrow A C T$ of 'executed actions' or 'action instantiations'. However, when possible, we simply talk about (labeled) actions in $A C$.

Three properties of actions that play a role in our policy system are described by the following functions:

- The observability function: obs $: A C \rightarrow P(\mathcal{G})$ describes which agents can observe which actions.

- The proof obligation function: po: $(A C \times \mathcal{G}) \rightarrow \Phi \cup\{\perp\}$ describes which policy an agent needs to justify the execution of an action. Here $\perp$ indicates that no policy is needed.

- The conclusion derivation function: concl : $(A C T \times \mathcal{G}) \rightarrow \Phi \cup\{\perp\}$, describes what policy can an agent deduce after observing an action. Here $\perp$ indicates that no policy can be deduced.

While the observability and proof obligation functions depend on executed actions (i.e. with identifiers), the conclusion derivation function is purely syntactical.

For our default actions creates $(a, d)$ and $\operatorname{comm}(a \Rightarrow b, \phi)$ we have:

$$
\begin{aligned}
\operatorname{obs}(\operatorname{creates}(a, d)) & =a \\
\text { obs }(\operatorname{comm}(a \Rightarrow b, \phi)) & =\{a, b\} \\
\operatorname{po}(\operatorname{creates}(a, d), a) & =\perp \\
\operatorname{po}(\operatorname{comm}(a \Rightarrow b, \phi), c) & =\perp \quad(a \neq c) \\
\operatorname{po}(\operatorname{comm}(a \Rightarrow b, \phi), a) & =a \text { says } \phi \text { to } b \\
\operatorname{concl}(\operatorname{creates}(a, d), a) & =a \text { owns } d \\
\operatorname{concl}(\operatorname{comm}(a \Rightarrow b, \phi), b) & =a \text { says } \phi \text { to } b \\
\operatorname{concl}(\operatorname{creates}(a, d), b) & =\perp \quad(b \neq a) \\
\operatorname{concl}(\operatorname{comm}(a \Rightarrow b, \phi), c) & =\perp \quad(c \neq b)
\end{aligned}
$$

A creation action by $a$ is observed by $a$ (1), while a communication between $a$ and $b$ is observed by both $a$ and $b$ (2). In other settings, there may also be other agents that observe these actions, e.g. a router standing in between $a$ and $b$. Agents do not need a policy for creating data (3) or receiving a transmission (4). However, sending a transmission does require a permission (5). If agent Alice creates a piece of data she becomes the owner of this data (6); any other agent can not deduce the ownership (8). If an agent receives a communication then the agent can conclude the corresponding says statement 7 . However, any other agent can not deduce any conclusion 9 .

Remark 1 Our communication comm $(a \Rightarrow b, \phi)$ models a point-to-point communication. We can easily model broadcasting, by introducing an action bcast $(a, \phi)$, and setting:

$$
\begin{aligned}
\text { obs }(\operatorname{bcast}(a, \phi)) & =\mathcal{G} \\
p o(\operatorname{bcast}(a, \phi), x) & = \begin{cases}\psi & (x=a) \\
\perp & \text { otherwise }\end{cases} \\
\operatorname{concl}(\operatorname{bcast}(a, \phi), x) & =\psi
\end{aligned}
$$

Where $\psi=\forall y$. a says $\phi$ to $y$.

Here, every agent can observe an action bcast $(a, \phi)$ and conclude that a has broadcasted $\phi$ i.e. said $\phi$ to everybody. Only a needs to justify this action. 


\subsection{The Proof System}

In the previous section we introduced the actions that agents can execute and the permissions that agents need to justify these actions, in form of policies. This section describes how agents perform this justification, i.e. how agents can build policies from (simpler) ones. The possibilities for constructing policies are given in the form of a derivation system or proof system for our policy language.

Each rule includes, besides the premises and conclusion, an agent $a$, called the context of the proof, indicating which agent is doing the reasoning. Our derivation system DER contains the standard predicate logic rules for introduction and elimination of conjunction, implication and universal quantification, together with the following rules:

$$
\begin{gathered}
\text { SAY } \frac{b \text { says } \phi \text { to } a}{\phi} a \\
\text { REFINE } \frac{\phi \rightarrow \psi \quad a \text { says } \phi \text { to } b}{a \text { says } \psi \text { to } b} a \\
\text { OBS_ACT } \frac{a c t \quad \operatorname{concl}(a c t, a) \neq \perp}{\operatorname{concl}(a c t, a)} a_{a} \\
\text { DER_POL } \frac{a \text { OWns } d_{1} \ldots a \text { owns } d_{n}}{\phi\left[\left\{d_{1}, \ldots, d_{n}\right\}\right]} a
\end{gathered}
$$

Rule (SAY) models delegation of responsibility. If agent $b$ says $\phi$ to $a$ then $a$ can assume $\phi$ to hold. (It is $b$ 's responsibility to show that it had permission to give $\phi$ to $a$, see Section 3.3 on accountability.) Although agent $a$ may use $\phi$ without further requirement, it does not mean that the agent must always do this. If Bob wants to do a specific sensitive action, he may only want to use communications that he 'trusts' in building his policy. For example, Bob would only trust and thus use a policy 'fire Charlie' if it is provided by his boss. If it is provided to him by coworker Alice, Bob will not use the policy, even though the responsibility of this policy would rest with Alice. In this setting, the problem of establishing and managing trust is orthogonal to the problem of obtaining policies: One could introduce a trust management system to assign a 'level of trust in a proof', and require that different levels of trust are established for different actions (see Section 6).

In our logic, Alice can refine her own policies, e.g. by adding extra conditions and obligations using the standard propositional rules. In addition, rule (REFINE) enables Alice to refine the policies she provides to other agents: if Alice is allowed to send some policy $\phi$ then she can also send any refinement of $\phi$. This notion of refinement isn't easily captured in natural deduction. The current notation is too general; without further restrictions it seems that $a$ can derive $\phi \rightarrow \psi$ for any (syntactically unrelated) $\phi$, provided she can assume $\psi$. Thus, we need to restrict the ability to use assumptions in the subproof of $\phi \rightarrow \psi$. In the formalization of our model, a double context sequent calculus, we can express this (see Appendix A].

Rule (OBS_ACT) links an action with its conclusion, given by the concl function. (OBS_ACT) applies when there is some conclusion (i.e. $\operatorname{concl}(a c t, a) \neq \perp$ ); e.g., from observing action $\operatorname{comm}(a \Rightarrow b, \phi) b$ derives $a$ says $\phi$ to $b$.

As we already mentioned, we design the logic in such a way that the owner of some data $d$ decides who is allowed to do which actions on $d$. In other words, an owner of some data $d$ is allowed to derive usage policies for $d$, targeted to any other agent. This is achieved by rule (DER_POL), which allows the creation of any usage policy for data which the agent owns. Non-owners can refine existing policies (e.g., policies they received), but cannot create new policies from scratch.

A derivation with these rules made by an agent is a proof.

Definition $2 A$ proof $\mathcal{P}$ of $\phi$ from agent a is a finite derivation tree such that: (1) each rule of $\mathcal{P}$ has a as subject; (2) each rule of $\mathcal{P}$ belongs to DER, (3) the root of $\mathcal{P}$ is $\phi$, and (4) each initial assumption is either an action, an obligation or a basic predicate.

We call conditions $\operatorname{cond}(\mathcal{P})$ of $\mathcal{P}$ the initial assumptions that are basic predicates, and actions act $(\mathcal{P})$ the initial assumptions which are observed unguarded actions (from rule $\left.\left(O B S \_A C T\right)\right)$. Finally, the multiset of initial assumptions that are guarded (by? and!) actions are called the obligations oblig $(\mathcal{P})$ of $\mathcal{P}$.

We now illustrate the usage of rules (REFINE) and (DER_POL) in the following example. 


$$
\begin{aligned}
& {[\operatorname{print}(b, d)]} \\
& {[\operatorname{rel}(d, x)]} \\
& \operatorname{print}(b, d) \quad \text { creates }(a, d)
\end{aligned}
$$

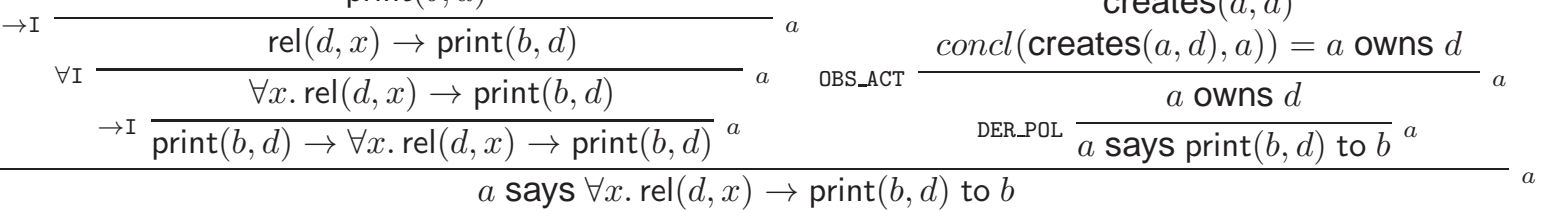

Table 1. Proof for Example 2

Example 2 (Policy Refinement) Suppose we have a predicate rel $(d, \bar{d})$, expressing whether two data objects are related: For instance, $d$ can be a review of a new product and $\bar{d}$ the press release announcing this product. Alice creates $d$ and wants to give a policy $\forall x$. rel $(d, x) \rightarrow \operatorname{print}(b, d)$ to Bob giving Bob permission to print the document as soon as a related object exists: Alice can build the policy allowing her to give this policy to Bob as shown in Table $\square$

\section{The Model}

We now introduce a model for our system, combining the different components of the previous sections. In our system, agents can execute and log actions. In addition to agents, an authority is also present. This authority may audit agents requiring justification for (some of) the agents actions.

\subsection{Logging actions}

Whenever an agent executes an action, it can also choose to log this action. Logged actions constitute evidences that can be used to demonstrate that an agent was allowed to perform a particular action. They are used during accountability auditing, in Section 3.3

Definition $3 \mathrm{~A}$ logged action is a triple lac $=\langle$ act,conds, obligs $\rangle$ consisting of an action act $\in A C$, a set of atomic predicates conds (the 'conditions'), and a set of labelled annotated actions obligs $\subset\{!, ?\} A C$ (the 'obligations'). The set of logged actions is denoted as LAC.

When logging an action, an agent can include supporting conditions which the environment certifies to be valid at the moment of execution of the action. This is recorded in the set of predicates conds. We do not model the environment explicitly but instead assume that the agent obtains a secure "package" of signed facts from the environment, represented in conds. As an example, one can think of the driver's license of Alice being checked to certify that she is over 21.

An agent can also include obligations in obligs in a logged action, which refers to other actions the agent did or promises to do. We abstract away from the details of expressing promises, and instead assume we have a way to check if actions have expired. For example, the agent may promise to pay within a day. Then a payment action needs to be done (and logged) within a day of logging this obligation. (Also see Section 3.3 )

Example 3 We continue with Example $\square 3$. Suppose that we introduce an action $\operatorname{drunk}(x, y)$ and a corresponding atomic predicate $\operatorname{drink}(x, y)$, with concl $(\operatorname{drunk}(x, y), x)=\perp$ and $p o(\operatorname{drunk}(x, y), x)=\operatorname{drink}(x, y)$. We also introduce an action paid $(x, y)$, with corresponding atomic predicate pay $(x, y)$, with $\operatorname{concl}(\operatorname{paid}(x, y), x)=p o(\operatorname{paid}(x, y), x)=\perp$.

A logged action lac $c_{\text {pay }}$ for payment is done first by $a$ :

$$
l a c_{\text {pay }}=\left\langle\operatorname{paid}_{0}(a, 10 \$), \emptyset, \emptyset\right\rangle
$$

Then, another logged action lac drunk for the action $\operatorname{drunk}_{1}(a$, beer $)$ is recorded:

$$
\begin{aligned}
l a c_{\text {drunk }}= & \left\langle\operatorname{drunk}_{1}(a, \text { beer }),\right. \\
& \left.\{\operatorname{age} 21(a), \text { alc }(\text { beer })\},\left\{\operatorname{paid}_{0}(a, 10 \$)\right\}\right\rangle
\end{aligned}
$$


The $\log$ of an agent $a$ is a finite sequence of logged actions. Note that it does not need to be $a$ who performed the actions, but of course $a$ has to observe an action to be able to log it. We say that agent $a \operatorname{logs}$ action act when $\langle a c t$, conds, obligs $\rangle$ is appended to the $\log$ of $a$, where conds is some set of conditions and obligs is some set of obligations.

We assume the following consistency properties of logging:

- An agent logs any action at most once, thus within an agent's log the logged actions are uniquely identified by the label (id) of the action.

- An agent can include the same obligation !actid at most once within the obligations of logged actions in its log. (an ?act $t_{i d}$ action, in contrast, may occur multiple time).

- An agent cannot $\log$ an expired action.

Notice that consistency of the log does not have to be checked at time of logging, it is sufficient to check it at time of auditing.

\subsection{The system model and state}

We are ready to introduce our system model.

Definition 4 A system is a 6-tuple:

$$
\langle\mathcal{G}, \Phi, A C T, \text { obs, concl, po }\rangle
$$

where $\mathcal{G}$ is a set of agents, $\Phi$ is the policy language, $A C T$ is a set of actions, and obs, concl and po are, respectively, the observability, conclusion and proof obligation functions.

A state $\mathcal{S}$ is the collection of logs of the different agents, i.e. a mapping from agents to logs. An agent who observes an action may choose to $\log$ this action. Thus by executing action act the system can make a transition from a state $\mathcal{S}$ to state $\mathcal{S}^{\prime}$, denoted $\mathcal{S} \stackrel{\text { act }}{\rightarrow} \mathcal{S}^{\prime}$ where $\mathcal{S}^{\prime}$ equals $\mathcal{S}$ except that the action act may have have been logged by any agent $a$ that can observe the action, $a \in$ obs $(a c t)$. An execution of the system consists of a sequence of transitions $\mathcal{S}_{0} \stackrel{\text { act }}{\rightarrow} \mathcal{S}_{1} \stackrel{\text { act }}{\rightarrow} \ldots \stackrel{\text { act }}{\rightarrow} \mathcal{S}_{n}$, starting with some initial state $\mathcal{S}_{0}$. The execution trace for this execution is act $a_{1} a_{2} \ldots a c t_{n}$. Actions logged by an agent can be also seen as a trace of actions, by projecting only the actions of each logged action. We denote that trace as $\mathcal{S}(a)$. Let $\preceq$ denote the subtrace relation $\left(t r_{1} \preceq t r_{2}\right.$ if each action of $t r_{1}$ is included in $t r_{2}$, and each time an action $a c t_{1}$ appears before $a c t_{2}$ in $t r_{1}$, the $a c t_{1}$ also appears before $a c t_{2}$ in $\left.t r_{2}\right)$. We have $\mathcal{S}(a) \preceq t r$.

Auditing Authority Agents may be audited by some authority, at some state $\mathcal{S}$. Intuitively, when some agent is about to be audited, an auditing authority is formed. This authority will audit the agent to find whether she is accountable for her actions. Let $t r$ be the sequence of actions executed from some initial state to $\mathcal{S}$, The evidence trace, denoted $\mathcal{E}$, contains all the actions that might be audited. Initially, $\mathcal{E}$ embeds $\mathcal{S}(a)$. However, $\mathcal{E}$ may also contain actions not in $\mathcal{S}(a)$ : They may be provided, for example, by some observing agents. However, we assume that given $\mathcal{S}(a)$ and other observed actions $S$, the authority can order properly the actions of $\mathcal{S}(a)$ and $S$ into $\mathcal{E}$, s.t. $\mathcal{E} \preceq t r$. Thus, in general $\mathcal{E}$ is a trace satisfying $\mathcal{S}(a) \preceq \mathcal{E} \preceq t r$.

\subsection{Accountability}

We now introduce notions of agent accountability, determined by some authority in possession of evidences. These definitions allow an authority to audit agents, to establish whether the agent was allowed to do the actions he did. In previous work [6], we defined several notions of agent and data accountability, but without checking for obligations nor conditions. We did not have logs of agents either. We now define accountability for logged actions, which we then extend to agent logs.

We first introduce justification proofs for logged actions. Intuitively, a justification proof is a proof of the policy required for the action (as given by function po), using only conditions and obligations that have been logged.

Definition 5 A proof $\mathcal{P}$ of $\phi$ from a is a justification (proof) of logged action $\langle$ act, conds, obligs $\rangle$ if:

- $p o(a c t, a)=\phi$ 
- The obligation in the proof are included in obligs; 'oblig $(\mathcal{P}) \subset$ obligs'. (Here multiple ?act in oblig $(\mathcal{P})$ may be assigned to the same ?act id but each occurrence of !act must have its own!act id in obligs.) ${ }^{1}$

- Each condition in the proof is in conds; $\operatorname{cond}(\mathcal{P}) \subseteq$ conds.

The set of all justifications is denoted by $\mathcal{J}$.

In general, there may be different justifications for an action. The justifications provided by the agent are modeled by a function $\operatorname{Pr}: \mathcal{G} \times L A C \rightarrow \mathcal{J} \cup\{\perp\}$. Here $\operatorname{Pr}(a,\langle a c t$, conds, obligs $\rangle)$ is either a valid justification of $\langle a c t$, conds, obligs $\rangle$, or it is $\perp$, indicating that the agent did not provide a justification.

Definition 6 (Logged Action Accountability) Agent a correctly accounts for logged action logact $=\langle$ act, conds, obligs $\rangle$ (in state $\mathcal{S})$, denoted $L A A(a$, logact), if:

- if po(act, $a) \neq \perp$ then $\operatorname{Pr}(a$, logact $) \neq \perp$, i.e. if needed a justification is provided

- if $o \in$ obligs has expired then $o \in \mathcal{S}(a)$, i.e. each obligation that has expired has been (executed and) logged.

- For each act $\in$ act $(\operatorname{Pr}(a$, logact $))$, a provides an id s.t. act ${ }_{i d}$ occurs in tr and $a \in$ obs $\left._{(a c t}{ }_{i d}\right)^{2}$

This definition introduces accountability for a single (logged) action. We now define accountability for any action and for all audited actions.

Definition 7 (Action Accountability) We say that agent a correctly accounts for (labeled) action act, denoted AA(a, act), if

- a has logged act as logact and LAA(a, logact) or

- a has not logged act and $L A A(a,\langle$ act $, \emptyset, \emptyset\rangle)$

We say agent a passes audit $\mathcal{E}$, written $A C C(a, \mathcal{E})$, if either $\mathcal{E}$ is the empty trace, or $\mathcal{E}=\mathcal{E}^{\prime}$.act with:

- $A A(a, a c t)$

- $A C C\left(a, \mathcal{E}^{\prime \prime}\right)$, with $\mathcal{E}^{\prime \prime}$ the correct ordered merge of $\mathcal{E}^{\prime}$ and newacts, all the new actions (i.e. not already in $\left.\mathcal{E}^{\prime}\right)$ given by the proofs in $A A(a, a c t)$.

The second case for action accountability explains why it can be in the interest of an agent to log its actions. As conditions may have changed, the agent can only rely on conditions if they have been logged at the time the action was executed. For example, if some action had a condition 'only execute between 4 p.m. and 4:30 p.m. on 12/10/2004', then that condition would only hold temporarily; If an agent executed the action and did not log it, during a later audit the agent could not provide a valid proof. The same holds for obligations: Only obligations logged with the action can be used in a proof of the action.

Claim 1 Let a be an agent and $\mathcal{E}$ an evidence trace. Then, checking $A C C(a, \mathcal{E})$ terminates.

Proof. Let $|t r|=n$, for some $n \geq 0$. Suppose $|\mathcal{E}|=m \leq n$. We show that each execution of $A C C(a, \mathcal{E})$ decreases $l$, with $l=n$ initially. After every execution of $A C C(a, \mathcal{E})$ as in Definition 7 at most $l-m$ evidences (the newacts) are added to $\mathcal{E}^{\prime \prime}$. Thus, $\left|\mathcal{E}^{\prime \prime}\right|=\left|\mathcal{E}^{\prime}\right|+(l-m)=m-1+(l-m)=l-1$. Hence, $A C C(a, \mathcal{E})$ terminates.

Honest Strategy A strategy for an honest agent $a$ to always be accountable is as follows. Before executing some action $a c t, a$ checks whether $p o(a c t, a)$ is derivable. If any obligation needs to be fulfilled, then the agent performs and then logs it. If any condition or obligation needs to be fulfilled, then the action act is also logged. Then, it follows from the definitions that:

Remark 2 (Accountability of honest agents) If agent a follows the honest strategy, then for any system execution and any auditing authority with evidence set $\mathcal{E}$, we have that $A C C(a, \mathcal{E})$ holds.

The proof follows immediately from Definitions 6 and 7

\footnotetext{
${ }^{1}$ interpreting as sets for ?act and as multisets for !act.

${ }^{2}$ We assume the authority can verify this.
} 
Recursive auditing We have, up to now, defined accountability of one particular agent in isolation. However, we may be interested in cross-verifying the actions of agents. We sketch an algorithm for recursive auditing of agents, which can be used by a potential auditing authority. The algorithm inputs $S_{0}$, a set of suspected agents, and $\mathcal{E}_{0}$, an initial evidence trace. Given $A C C(a, \mathcal{E})$, we write $E(A C C(a, \mathcal{E}))$ to denote the set of new actions appearing in the given proofs (the newacts in Definition [7], and $A(A C C(a, \mathcal{E}))$ the corresponding set of agents appearing in these actions for which the proof obligation is not bottom, i.e. $p o(\cdot, \cdot) \neq \perp$.

Algorithm 1 (Recursive Auditing) Inputs: $S_{0}$ and $\mathcal{E}_{0}$. Outputs: true if audited agents are accountable, false otherwise.

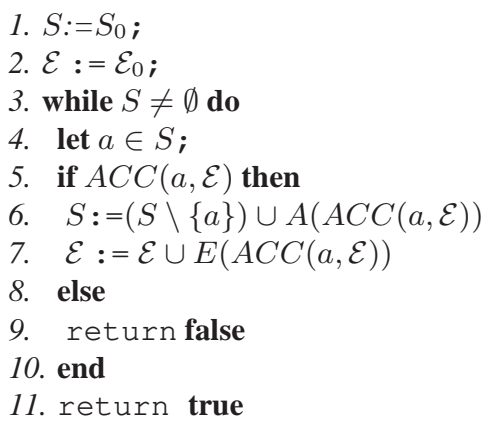

Claim 2 Algorithm पterminates.

Proof. Similar to the proof of Claim 1

Claim 3 In line 4 of of Algorithm $\square$ the order in which the agents are chosen does not matter.

Proof (sketch). Follows from the fact that proofs are fixed on beforehand, as given by function $\operatorname{Pr}: \mathcal{G} \times L A C \rightarrow \mathcal{J} \cup\{\perp\}$, and do not depend on knowing whether other agents are being audited or not. (Intuitively, this models the fact that agents can not change their proofs on the fly, depending on whether other agents are being audited.)

\section{Formalization}

We have implemented the proof system and checking of the audit logic in Twelf [13], which is an implementation of the Edinburgh Logical Framework [12]. Research in proof-carrying code [10] has shown that Logical Framework (LF) provides a suitable notation for proofs to be sent and checked by a recipient. In type theories proof checking reduces to type checking and the LF proof checker is as simple as a programming language type checker (see also Section 5 .

Here we only mention the most important features of the implementation. In the appendices we have included both a more abstract representation (common for a sequent calculus), as well as the complete Twelf-file.

Audit Logic Implementation We first declare the types of the object logic, the atomic predicates and the actions. Then we present the proof rules and we finish with an example of a complete proof.

Types In Twelf, a metalogic type is of type type. For object logic types, we use the type $t p$. The meta-logic function $->$ goes from type to type or kind (type has type kind). Agents, data, actions and policies are declared as tps. Finally, when declaring the rules for the object logic the tm type constructor is used, which casts arguments from tp to type. Summarizing:

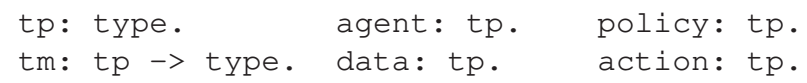

Policies Recall that policies are formed with says and owns and for example scenario-specific atomic predicates like print:

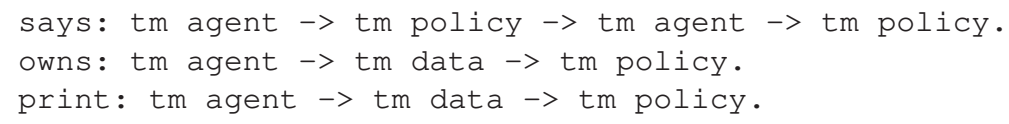


For instance, (print a d) is a policy stating that agent a has permission to print document d. Policies can also be formed using the usual propositional connectives and universal quantification ${ }^{3}$ (except for negation and disjunction); to model the different instances of implication in the policy language (see definition 1), we declare separate instances for use-onceobligations and use-many-obligations:

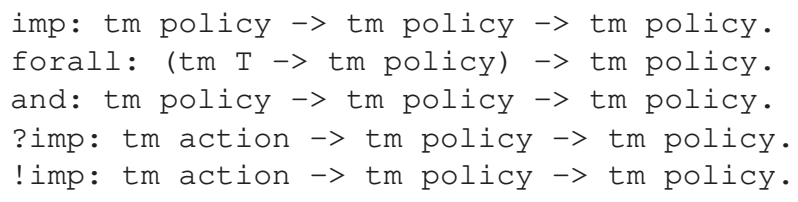

Below we assume that the connectives imp, and, ? imp, ! imp are declared as infix-operators.

Actions The actions creates and comm have the following types:

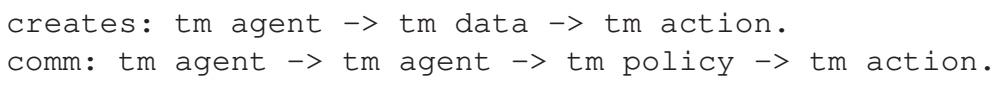

The conclusion derivation function describes the policies the agent can deduce from some action.

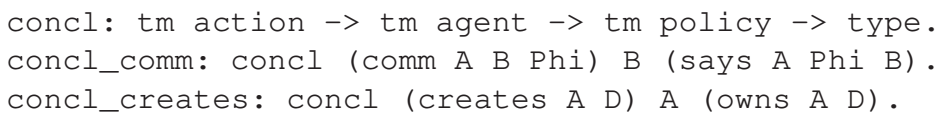

In Twelf, symbols with a leading capital are variables. Their type can often be left unspecified, as Twelf expands them to the most general type.

Proof derivation To model local proofs (with respect to agents), we use sequent calculus formulas of the form $\Gamma$; $\Delta \vdash{ }_{A} \Phi$, indicating that agent $A$ can deduce the policy $\Phi$ from premises $\Gamma$ and $\Delta$. Here $\Gamma$ is an unrestricted context and $\Delta$ a linear context. More precisely, $\Gamma$ is a sequent of policies, actions and ?obligations, while $\Delta$ contains only !obligations. We formalize this with

entail: tm agent $\rightarrow$ list nonlin $\rightarrow$ list lin $\rightarrow$ tm policy $\rightarrow$ type.

where list $\mathrm{T}$ is the type for lists of type $\mathrm{T}$. The type nonlin is like a disjoint union (policy, action, action) by the following definitions:

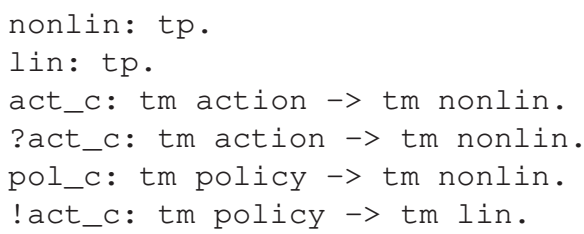

Rules The rules SAY and REFINE are defined as follows:

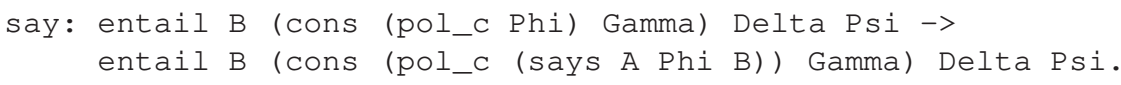

The OBS_ACT rule works as follows. If an agent $A$ can conclude the policy $\Phi$ by observing action act, then she can deduce $\Phi$ from any set $\Gamma$ containing act. In formula $\Gamma$, act; $\Delta \vdash_{A} \Phi$, in Twelf:

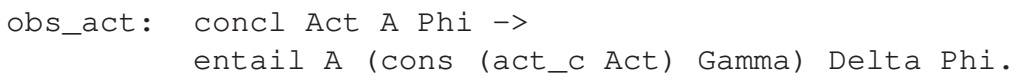

The REFINE rule implements the possibility of refining a policy, $\phi$ to $\psi$ and saying $\psi$, if one has permission to say $\psi$. Refinement in this sense is expressed using empty sequents:

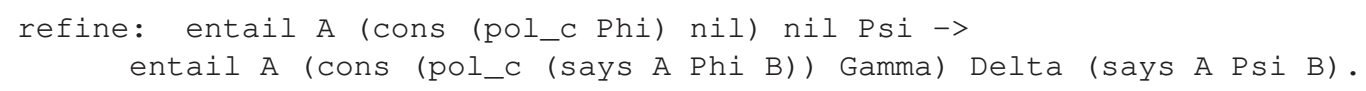

\footnotetext{
${ }^{3}$ Quantification over policies is not allowed, so we only instantiate the $\forall$-left and -right rules for the types agent and data.
} 
The meaning of the DER_POL rule is that any formula can be derived, as long as, for all data affected by it, ownership is proven. To this end we define a relation on a non-linear sequent $\Gamma$ and a policy $\phi, o p[\Gamma ; \phi]$, that only holds iff all data affected by $\phi$, occur in some owns-predicate in $\Gamma$.

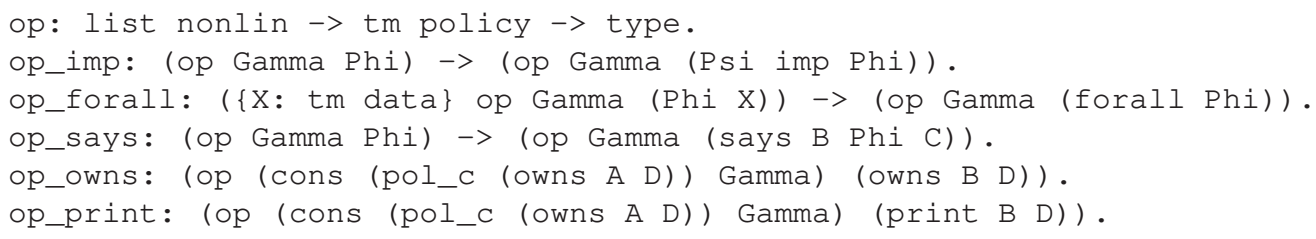

Now the DER_POL rule is defined as follows:

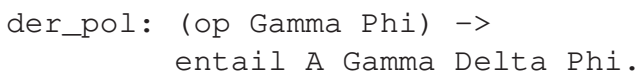

Please note that the structural rules and the logical rules for conjunction, implication and universal quantification over agents and data are omitted here, but they can be found in the appendices.

Example 4 (Formalization of Example 2) Recall that Alice creates a document d (a review of a product) and communicates a policy, to Bob, that gives Bob permission to print d 'as soon as a related object exists'. Below Alice justifies that she can do so. First we declare the scenario-specific objects and predicates.

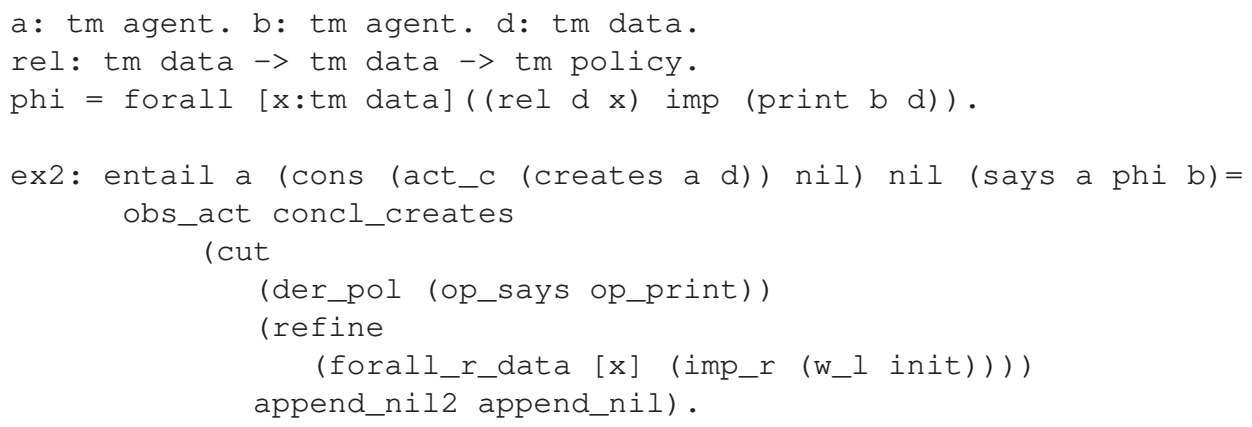

This theorem has been checked by Twelf. The proof goes along the same lines as previously in Table 1

The lhs can be expressed as $\gamma ; \delta \vdash_{a}$ ( $a$ says $\phi$ to $b$ ), where $\phi$ is $\forall x$. rel $(d, x) \rightarrow \operatorname{print}(\mathrm{b}, \mathrm{d})$ and $\gamma$ only contains the creates action, while $\delta$ is empty. In the proof above, imp_r is the imp-right rule, forall_r_data is the $\forall-r i g h t$ rule for quantification over data, $\mathrm{w}_{-} 1$ and init are weakening-left and the initial sequent axiom for the non-linear sequent.

\section{Related Work}

There is a wide body of literature on logics in Access Control (see the survey by Abadi [1]). Here, we mention some of the proposals. Binder [7] is a logic-based security language based on Datalog Binder includes a special predicate, says, used to quote other agents. Binder's says differs in two aspects from our construct: First, ours includes a target agent (see Section 2.4; Second, when importing (i.e. communicating a policy in our setting) a clause in Binder, care must be taken to avoid nested says, since it may introduce difficulties in their setting. More related to our auditing by means of proofs, Appel and Felten [2] propose the Proof-Carrying Authentication framework (PCA), also implemented in Twelf (see Section 4). Differently from our work, PCA's language is based on a higher order logic that allows quantification over predicates. Also, their system is implemented as an access control system for web servers, while in our case we focus on a-posteriori auditing.

BLF [19] is an implementation of a Proof-Carrying-Code framework that uses both Binder and Twelf, which however focuses on checking semantic code properties of programs.

Sandhu and Samarati [16] give an account of access control models and their applications. Bertino et al. [4] propose a framework for reasoning on access control models, in which authorization rules treat the core components Subjects, Objects and Privileges. Sandhu and Park [11] take a different approach with their UCON-model, in which the decision is modelled as a reference monitor that checks the 3 components: ACL, Conditions and Obligations. This reflects much the separation also made by us. Obligations and conditions are also prominent in directives on privacy and terms of use in DRM. The concept of purpose of an action is not used by us, but is used in the privacy languages P3P and E-P3P [3]. Unlike our policy language, E-P3P allows the use of negation, which requires special care to avoid problems in a distributed setting. 


\section{Conclusions and Future Work}

We have presented a flexible usage policy framework which enables expressing and reasoning about policies and user accountability. Enforcement of policies is difficult (if not impossible) in the highly distributed setting we are considering. Instead, we propose an auditing system with best-effort checking by an authority depending on the power of the authority to observe actions. A notion of agent accountability is introduced to express the proof obligation of an agent being audited.

Our obligations cover pre- and post-obligations ([15]) but not yet ongoing obligations. The setup does, with an adaption of the definitions of accountability, seem to provide the means to include this type of obligations. Obligations are 'use once', e.g. !pay $(\$ 10)$ or 'use as often as wanted' ?pay $(\$ 10)$.

Our proof system has been implemented using the proof checker Twelf. The agents develop proofs using this implementation. Likewise, the implementation allows an authority to check the agents' proofs.

In our system, we include a powerful rule which allows delegating any policy to any other agent. Agent Alice may only want to use a policy from Bob if she (i) knows Bob, (ii) authenticates Bob, and (iii) trusts Bob. All these issues are (intentionally) abstracted away in our approach, as they seem to be orthogonal to our aims. For example, in (iii), the required level of trust may depend on the policy provided by Bob or on the way Alice is going to use the policy. There, a distributed trust management system (e.g. [9]) could be employed to obtain the required level of trust.

In the work of Samarati et.al. [14], a discussion about decentralized administration is presented. Specially, the revocation of authorizations is addressed. This is acomplex problem, which occurs as a consequence of the delegation of privileges. One could model revocation of policies by adding a special flag plus a corresponding check in a policy. However, checking whether a flag is set in another agent's environment is not realistic in our highly distributed setting. Further research is needed to find a both practical and realistic way to include rights revocation.

Our implementation only covers proof checking. Revisiting Figure1 we find that arrows covering policy communications and logging are not yet implemented. Our framework requires several properties for each of the different modules (e.g. secure logging and non-repudiable communications). Certainly, these properties need cryptography to be realized securely. We regard as future work the rigorous cryptographic definition of these properties, along with the accompanying cryptographic constructions.

Acknowledgements The research presented in this paper is conducted within the PAW project (funded by Senter-IOP and TNO), the Inspired Project and the NWO Account Project.

\section{References}

[1] M. Abadi. Logic in access control. In Proc. 8th Annual IEEE Symposium on Logic in Computer Science (LICS), pages 228-233. IEEE Computer Society Press, 2003.

[2] A. W. Appel and E. W. Felten. Proof-carrying authentication. In G. Tsudik, editor, Proc of the 6th Conference on Computer and Communications Security. ACM Press, 1999.

[3] P. Ashley, S. Hada, G. Karjoth, and M. Schunter. E-p3p privacy policies and privacy authorization. In P. Samarati, editor, Proc. of the ACM workshop on Privacy in the Electronic Society (WPES 2002), pages 103-109. ACM Press, 2002.

[4] E. Bertino, B. Catania, E. Ferrari, and P. Perlasca. A logical framework for reasoning about access control models. ACM Transactions on Information and System Security (TISSEC), pages 71-127, 2003.

[5] C. N. Chong, R. Corin, S. Etalle, P. H. Hartel, W. Jonker, and Y. W. Law. LicenseScript: A novel digital rights language and its semantics. In K. Ng, C. Busch, and P. Nesi, editors, 3rd Int. Conf. on Web Delivering of Music (WEDELMUSIC), pages 122-129. IEEE Computer Society Press, 2003.

[6] R. Corin, S. Etalle, J. I. den Hartog, G. Lenzini, and I. Staicu. A logic for auditing accountability in decentralized systems. In T. Dimitrakos and F. Martinelli, editors, Proc. of the second IFIP Workshop on Formal Aspects in Security and Trust (FAST), volume 173, page to appear. Springer Verlag, 2004.

[7] John DeTreville. Binder, a logic-based security language. In Proceedings of the IEEE Symposium on Research in Security and Privacy, pages 105-113. IEEE Computer Society Press, 2002. 
[8] S. Jajodia, P. Samarati, V. S. Subrahmanian, and E. Bertino. A unified framework for enforcing multiple access control policies. In J. Peckham, editor, SIGMOD 1997, Proc. International Conference on Management of Data, pages 474485. ACM Press, 1997.

[9] N. Li, J. Mitchell, and W. Winsborough. Design of a role-based trust-management framework. In Proc. of the IEEE Symposium on Research in Security and Privacy, pages 114-130. IEEE Computer Society Press, 2002.

[10] G. C. Necula. Compiling with Proofs. PhD thesis, School of Computer Science, Carnegie Mellon University, Pittsburgh, PA, 1998.

[11] J. Park and R. Sandhu. Towards usage control models: Beyond traditional access control. In R. Sandhu, editor, Proc. of the Seventh ACM Symposium on Access Control Models and Technologies (SACMAT-02), pages 57-64. ACM Press, 2002.

[12] F. Pfenning. Logic programming in the LF logical framework. In G. Huet and G. Plotkin, editors, Logical Frameworks, pages 149-181. Cambridge University Press, 1991.

[13] F. Pfenning and C. Schürmann. System description: Twelf - A meta-logical framework for deductive systems. In H. Ganzinger, editor, Proc. of the 16th International Conference on Automated Deduction (CADE-16), pages 202-206. Springer-Verlag, 1999.

[14] P. Samarati and S. De Capitani di Vimercati. Access control: policies, models, and mechanisms. In R. Focardi and R. Gorrieri, editors, Foundations of Security Analysis and Design, LNCS, volume 2171, pages 137-196. SpringerVerlag, 2001.

[15] R. Sandhu and J. Park. Usage control: A vision for next generation access control. In V. Gorodetsky, L. J. Popyack, and V. A. Skormin, editors, Proc. Second International Workshop on Mathematical Methods, Models, and Architectures for Computer Network Security MMM-ACNS, volume 2776 of LNCS, pages 17-31. Springer-Verlag, 2003.

[16] R. Sandhu and P. Samarati. Access control: Principles and practice. IEEE Communications Magazine, 32(9):40-48, 1994.

[17] W3C. A p3p preference exchange language 1.0 (appel1.0). www.w3.org/TR/P3P-preferences, 2002.

[18] X. Wang, G. Lao, T. De Martini, H. Reddy, M. Nguyen, and E. Valenzuela. XrML: eXtensible rights markup language. In M. Kudo, editor, Proc. 2002 ACM workshop on XML security (XMLSEC-02), pages 71-79. ACM Press, 2002.

[19] N. Whitehead, M. Abadi, and G. C. Necula. By reason and authority: A system for authorization of proof-carrying code. In Proc. of the 17th Computer Security Foundations Workshop, pages 236-250. IEEE Computer Society Press, 2004.

\section{A The Sequent calculus for the Audit Logic}

In Twelf we implemented a double context sequent calculus. To explain the Twelf-code more clearly, we start by giving the (for sequent calculi) common representation of our system. In the formalization of our model we have in general $\langle$ context $\rangle$; $\langle$ linear context $\rangle \vdash_{A}$ policy, where $A$ is the agent doing the reasoning, but below we can write shortly $\Gamma ; \Delta \vdash \phi \quad$ (because rules regard only one agent-context). The contexts are sequents of linear and nonlinear propositions that serve as premises for the conclusion (right of the $\vdash$ sign). Now, in the Twelf code we declare 6 types of objects: agent, data, action, policy, nonlin, lin and a type list. In the sequel Greek and Roman letters denote variables that range over them. The type of the variables is often left implicit, so to distinguish the different types we use; A, B, C to denote agents, $\Gamma, \Gamma^{\prime}$ for lists of type nonlin, $\Delta, \Delta^{\prime}$ for lists of type lin, $\gamma_{0}$ and $\delta_{0}$ for the empty lists of type lin and nonlin. Policies are denoted with $\phi, \phi^{\prime}, \psi$ and $\xi, \xi^{\prime}$ denote actions. To express append and construct operators for lists we just write a comma. And finally $\rightarrow, \wedge, \forall$ denote the policy connectives for implication, conjunction and universal quantification. We use ?imp and !imp for the implication operators on obligations. Finally, in the non-linear context, we mix policy formulas (introduced by the standard logical rules), with actions (introduced by obs_act) and use-many-obligations (introduced with ?imp ). In Twelf each of these is mapped to the type nonlin. This context is thus like a list of the disjoint union of policy, action and action. 
Below we only mention the main inference rules. For the type declarations and the definitions of lists and so on we refer to the Twelf code in the appendix.

First our pivotal rules. Here we mention the $A$ in $\vdash_{A}$ because the agent appears explicitly in the formulas.

$$
\begin{aligned}
& \operatorname{SAY} \frac{\Gamma, \phi ; \Delta \vdash_{\mathrm{A}} \psi}{\Gamma, \operatorname{says}(\mathrm{B}, \phi, \mathrm{A}) ; \Delta \vdash_{\mathrm{A}} \psi} \\
& \text { OBS_ACT } \frac{\Gamma, \operatorname{concl}(\xi, \mathrm{A}) ; \Delta \vdash \psi}{\Gamma, \xi ; \Delta \vdash_{\mathrm{A}} \psi} \\
& \operatorname{REFINE} \frac{\gamma_{0}, \phi ; \delta_{0} \vdash_{\mathrm{A}} \psi}{\Gamma, \operatorname{says}(\mathrm{A}, \phi, \mathrm{B}) ; \Delta \vdash_{\mathrm{A}} \operatorname{says}(\mathrm{A}, \psi, \mathrm{B})} \\
& \text { DER_POL } \frac{\mathrm{op}[\Gamma ; \psi]}{\Gamma ; \Delta \vdash_{\mathrm{A}} \psi}
\end{aligned}
$$

Note the apparent difference with for instance the SAY-rule in natural deduction style in Section 2.4 It seems to have been turned upside-down; this is done to both keep the sub-formula-property, and preserve the redundancy of the cut-rule ${ }^{4}$.

The OBS_ACT-rule uses the conclusion derivation function, which is defined as:

$$
\begin{aligned}
& \text { concl_creates : } \operatorname{concl}(\operatorname{creates}(A, D), A)=\operatorname{owns}(A, D) \text {. } \\
& \text { concl_comm : } \operatorname{concl}(\operatorname{comm}(A, B, \phi), B)=\operatorname{says}(A, \phi, B) .
\end{aligned}
$$

A few words on the REFINE-rule: The intention of the empty sequents is to prevent that an agent uses a fact $\psi$ to derive $\phi \rightarrow \psi$ for an otherwise unrelated $\phi$. In formulas: $\Gamma, \psi \vdash(\phi \rightarrow \psi)$ holds for any $\Gamma$ and $\phi$. Then with just the permission to say $\phi$, the agent can say $\psi$, which is of course not the meaning of refining the policy $\phi$. This subtlety is not easily expressed in natural deduction rules, but in the sequent calculus this is done straightforwardly with the empty sequent $\gamma_{0}$. With this restriction in place only syntactically related policies can be used, for example for $\psi=(\xi \rightarrow \phi)$ or $\psi=\forall \phi$. While, if there exists such a relation for two (syntactically unrelated) predicates, then it should be expressed by adding a rule for it: $\gamma_{0}$, write $(A, D) \vdash \operatorname{read}(A, D)$.

Finally, the DER_POL-rule expresses that if a policy sets permissions on data $D_{1}, \ldots, D_{n}$ and each of the $D_{1}, \ldots, D_{n}$ is owned by $A$ then $A$ can derive that policy. The notion of dataset of a policy in the article, is thus formalized a bit stronger here with the notion of active dataset, being the data that is actually affected by the policy. To this end we defined a relation on $\Gamma$ and $\phi: \circ p[\Gamma ; \phi]$ that holds iff all data affected by $\phi$, occur in some owns-predicate in $\Gamma$. The following rules make up its definition:

$$
\begin{aligned}
& \text { op_imp } \frac{\mathrm{op}[\Gamma ; \phi]}{\mathrm{op}[\Gamma ;(\psi \rightarrow \phi)]} \\
& \text { op_forall } \frac{\mathrm{op}[\Gamma ; \phi(\mathrm{X})]}{\mathrm{op}[\Gamma ; \forall \phi]}(\mathrm{X} \text { a fresh constant }) \\
& \text { op_says } \frac{\mathrm{op}[\Gamma ; \phi]}{\mathrm{op}[\Gamma ; \operatorname{says}(\mathrm{B}, \phi, \mathrm{C})]} \\
& \text { op_owns } \frac{\mathrm{op}[\Gamma, \text { owns }(\mathrm{A}, \mathrm{D}) ; \text { owns(B, D)] }}{\text { op_print } \frac{\mathrm{op}[\Gamma, \text { owns }(\mathrm{A}, \mathrm{D}) ; \operatorname{print}(\mathrm{B}, \mathrm{D})]}{}}
\end{aligned}
$$

\footnotetext{
${ }^{4} \mathrm{~A}$ full proof of cut-admissibility and other meta-theorems are future work
} 
Where the last one is to show a scenario-specific predicate. Now we present the standard rules for the sequent calculus:

$$
\begin{aligned}
& \text { init } \overline{\Gamma, \phi ; \Delta \vdash \phi} \\
& \operatorname{cut} \frac{\Gamma ; \Delta \vdash \phi \quad \Gamma^{\prime}, \phi ; \Delta^{\prime} \vdash \psi}{\Gamma, \Gamma^{\prime} ; \Delta, \Delta^{\prime} \vdash_{\mathrm{A}} \psi} \\
& \operatorname{and} \_11 \frac{\Gamma, \phi ; \Delta \vdash \phi^{\prime}}{\Gamma,(\phi \wedge \psi) ; \Delta \vdash \phi^{\prime}} \\
& \operatorname{and} \_12 \frac{\Gamma, \psi ; \Delta \vdash \phi^{\prime}}{\Gamma,(\phi \wedge \psi) ; \Delta \vdash \phi^{\prime}} \\
& \text { and } \_\frac{\Gamma ; \Delta \vdash \phi \quad \Gamma^{\prime} ; \Delta^{\prime} \vdash \psi}{\Gamma, \Gamma^{\prime} ; \Delta, \Delta^{\prime} \vdash(\phi \wedge \psi)} \\
& \text { imp_l } \frac{\Gamma ; \Delta \vdash \phi \quad \Gamma^{\prime}, \phi^{\prime} ; \Delta^{\prime} \vdash \psi}{\Gamma, \Gamma^{\prime},\left(\phi \rightarrow \phi^{\prime}\right) ; \Delta, \Delta^{\prime} \vdash \psi} \\
& \operatorname{imp} \_\frac{\Gamma, \phi ; \Delta \vdash \psi}{\Gamma ; \Delta \vdash(\phi \rightarrow \psi)}
\end{aligned}
$$

We need similar left and right rules for the !imp and ?imp operators. Recall that use-many obligations go into the unrestricted context, while use-once obligations go into the linear context. Here are the two pairs:

$$
\begin{aligned}
& \operatorname{limp} \_\frac{\Gamma ; \Delta \vdash(\xi \operatorname{limp} \phi)}{\Gamma ; \Delta, \xi \vdash \phi} \\
& \text { !imp_r } \frac{\Gamma ; \Delta \vdash \phi}{\Gamma ; \Delta \vdash(\xi \operatorname{limp} \phi)} \\
& \text { ?imp_l } \frac{\Gamma ; \Delta \vdash(\xi \text { ?imp } \phi)}{\Gamma, \xi ; \Delta \vdash \phi} \\
& \text { ?imp } r \frac{\Gamma ; \Delta \vdash \phi}{\Gamma ; \Delta \vdash(\xi ? \operatorname{imp} \phi)}
\end{aligned}
$$

In Twelf we coded twice the following $\forall$-left and $\forall$-right rules; one for agents, one for data. But not for actions or policies.

$$
\begin{aligned}
& \text { forall_l } \frac{\Gamma, \phi(\mathrm{X}) ; \Delta \vdash \psi}{\Gamma, \forall \phi ; \Delta \vdash \psi} \\
& \text { forall_r } \frac{\Gamma ; \Delta \vdash \phi(\mathrm{X})}{\Gamma ; \Delta \vdash \forall \phi}(\mathrm{X} \text { a fresh constant })
\end{aligned}
$$

Finally, we have the structural rules for the unrestricted and the linear contexts. The linear context doesn't allow for 
contraction.

$$
\begin{aligned}
& \text { w_l } \frac{\Gamma ; \Delta \vdash \phi}{\Gamma, \psi ; \Delta \vdash \phi} \\
& \text { w_l_act } \frac{\Gamma ; \Delta \vdash \phi}{\Gamma ; \Delta, \xi \vdash \phi} \\
& \text { contr_l } \frac{(\Gamma, \phi), \phi ; \Delta \vdash \psi}{\Gamma, \phi ; \Delta \vdash \psi} \\
& \text { perm_l } \frac{\Gamma, \phi, \phi^{\prime}, \Gamma^{\prime} ; \Delta \vdash \psi}{\Gamma, \phi^{\prime}, \phi, \Gamma^{\prime} ; \Delta \vdash \psi} \\
& \text { perm_act } \frac{\Gamma ; \Delta, \xi, \xi^{\prime}, \Delta^{\prime} \vdash \phi}{\Gamma ; \Delta, \xi^{\prime}, \xi, \Delta^{\prime} \vdash \phi}
\end{aligned}
$$

Which wraps up the list of rules. We now show some examples of proofs in this notation.

\section{A.1 Example: Creating and consuming a policy}

Let's draw the proof-tree of example $2 \mathrm{a}$ in this notation. We use abbreviations in this example:

$$
\begin{aligned}
& \phi=\forall \psi \quad \psi(x)=\left(\psi_{1}(x) \rightarrow \psi_{2}\right) \\
& \psi_{1}(x)=\operatorname{rel}(d, x) \quad \psi_{2}=\operatorname{print}(b, d) \\
& \gamma=\gamma_{0}, \text { owns }(a, d) \quad \gamma^{\prime}=\gamma_{0}, \psi_{1}\left(d^{\prime}\right)
\end{aligned}
$$

In words: Agent $a$ proves that she can say policy $\phi$ to agent $b$.

$$
\begin{aligned}
& \text { op_print } \overline{\mathrm{op}\left[\gamma ; \psi_{2}\right]}
\end{aligned}
$$

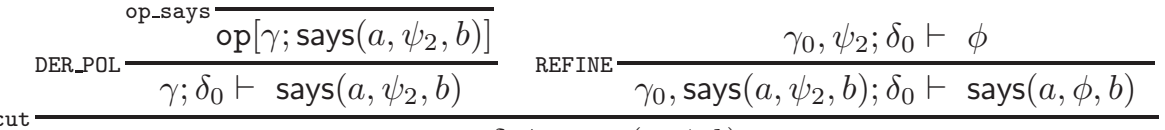

$$
\begin{aligned}
& \text { OBS_ACT } \frac{\gamma ; \delta_{0} \vdash \operatorname{says}(a, \phi, b)}{\gamma_{0}, \text { creates }(a, d) ; \delta_{0} \vdash \operatorname{says}(a, \phi, b)}
\end{aligned}
$$

Please note that the use of both the REFINE and the DER_POL-rule, and thus the cut-rule, is only done to stay close to the natural deduction style proof given earlier in the article (see Table 1). In fact, by using only the DER_POL-rule one could derive the same permission. The premise of the REF INE-rule is easily proven:

$$
\text { forall_r } \frac{\text { imp_r } \frac{\text { w_l } \frac{\text { init } \frac{}{\gamma_{0}, \psi_{2} ; \delta_{0} \vdash \psi_{2}}}{\gamma_{0}, \psi_{2}, \psi_{1}(y) ; \delta_{0} \vdash \psi_{2}}}{\gamma_{0}, \psi_{2} ; \delta_{0} \vdash\left(\psi_{1}(y) \rightarrow \psi_{2}\right)}}{\gamma_{0}, \psi_{2} ; \delta_{0} \vdash \forall \psi}
$$

Now agent $b$, who receives the mentioned policy, proves that he has the permission to print - using the evidence of the communication by $a$ and the assertion on $d^{\prime}$ (inside $\gamma^{\prime}$ ) which is asserted by $b$ 's environment.

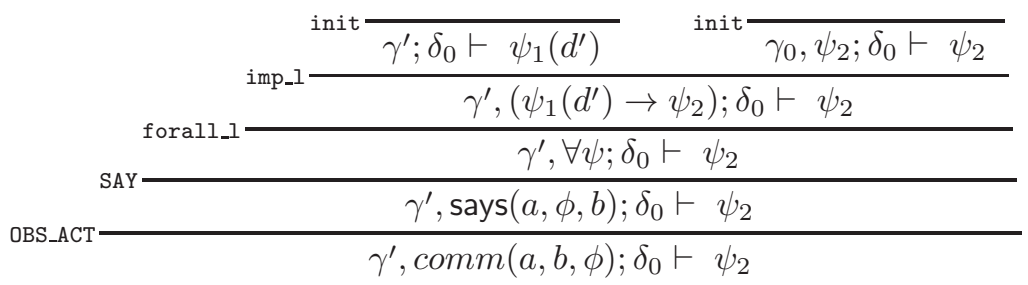




\section{A.2 Example: Fulfilling use-once obligations}

Finally let's see a policy with !imp in it. The drink-beer example is fine. Suppose the bartender, $a$, has communicated the policy $\phi$ to a customer, $b$. The customer has to prove $\operatorname{po}(\operatorname{drunk}(b$, beer $))=\operatorname{drink}(b$, beer $)$. The abbreviations are:

$$
\begin{aligned}
& \phi=\forall \psi \quad \psi(x)=\left(\psi_{1}(x) ! \operatorname{imp} \psi_{2}(\mathrm{x})\right) \\
& \psi_{1}(x)=\operatorname{paid}(x, 5 \$) \quad \psi_{2}(x)=\operatorname{drink}(x, \text { beer }) \\
& \xi=\operatorname{comm}(a, b, \phi) \quad \xi^{\prime}=\operatorname{paid}(b, 5 \$)
\end{aligned}
$$

Now agent $b$ uses the evidence of the communication and the obligation paid $(b, 5 \$)$ to prove that he is allowed to drink one beer having paid $5 \$$.

$$
\text { OBS_ACT } \frac{\text { SAY } \frac{\text { forall__ } \frac{\text { init } \frac{}{\gamma_{0}, \psi(b) ; \delta_{0} \vdash\left(\psi_{1}(b) ! \text { imp } \psi_{2}(\mathrm{~b})\right)}}{\gamma_{0}, \forall \psi ; \delta_{0}, \xi^{\prime} \vdash \psi_{2}(b)}}{\gamma_{0}, \operatorname{says}(a, \forall \psi, b) ; \delta_{0}, \xi^{\prime} \vdash \psi_{2}(b)}}{\gamma_{0}, \xi ; \delta_{0}, \xi^{\prime} \vdash \psi_{2}(b)}
$$

\section{B Twelf Code}

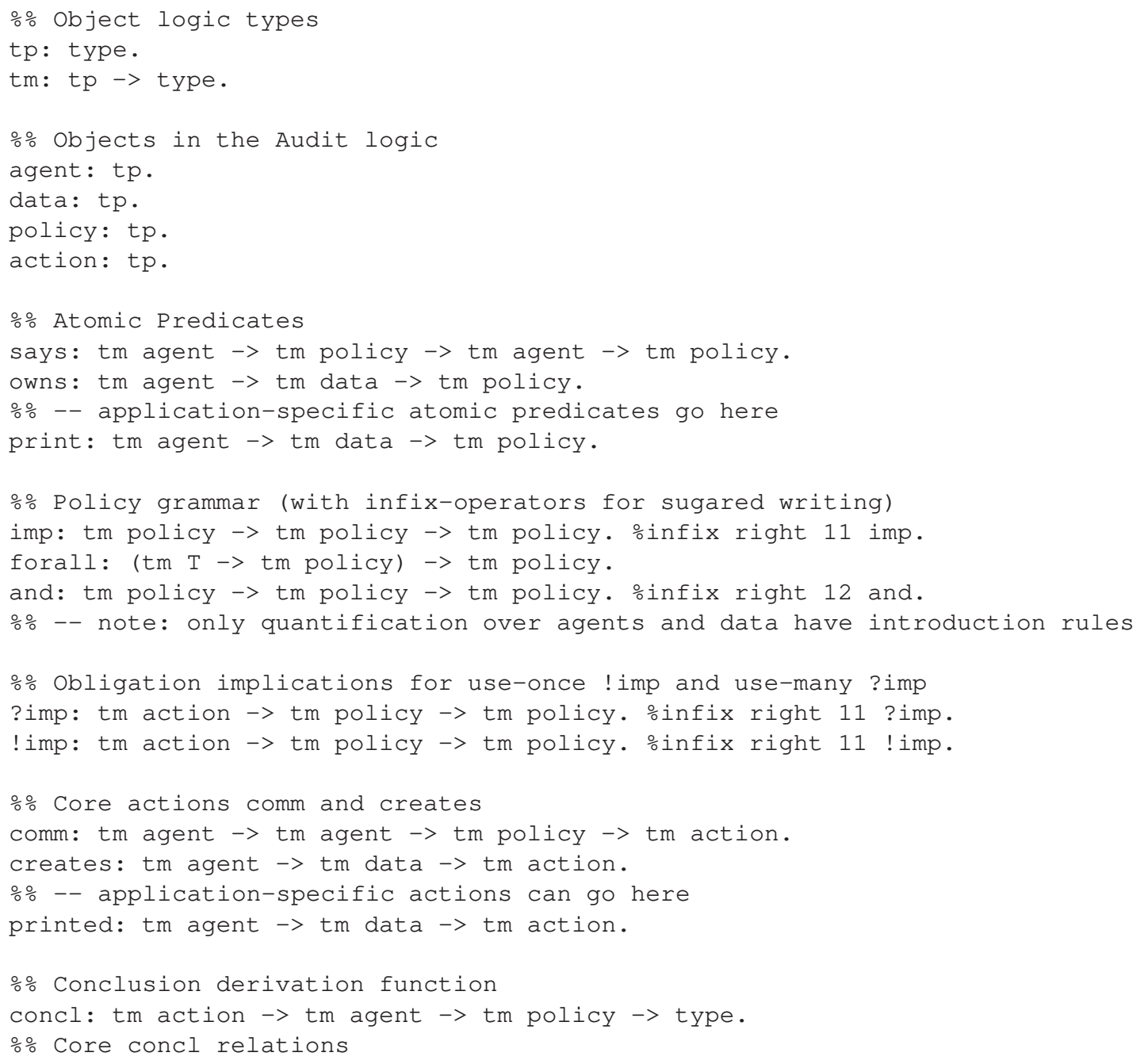




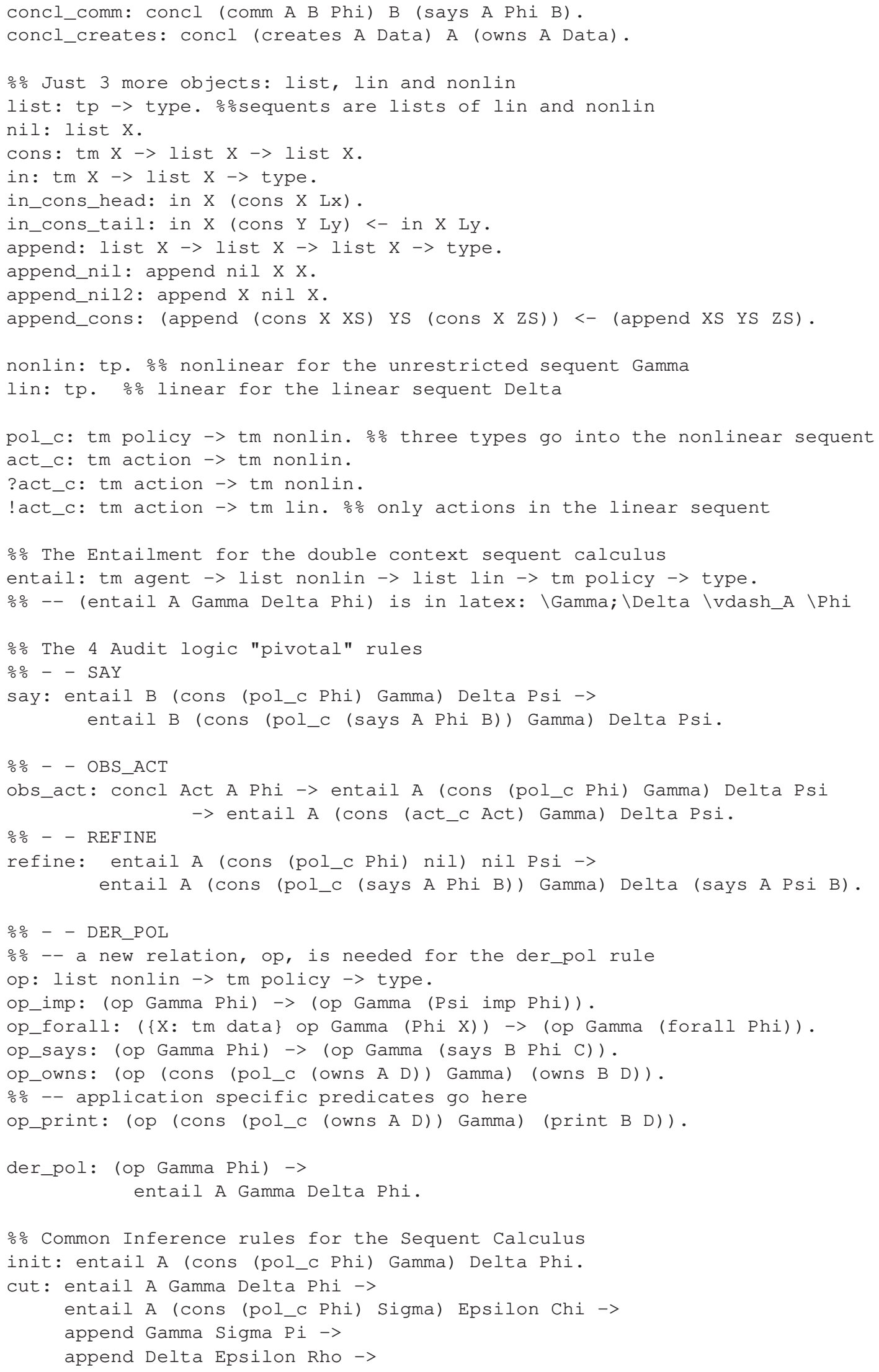


entail A Pi Rho Chi.

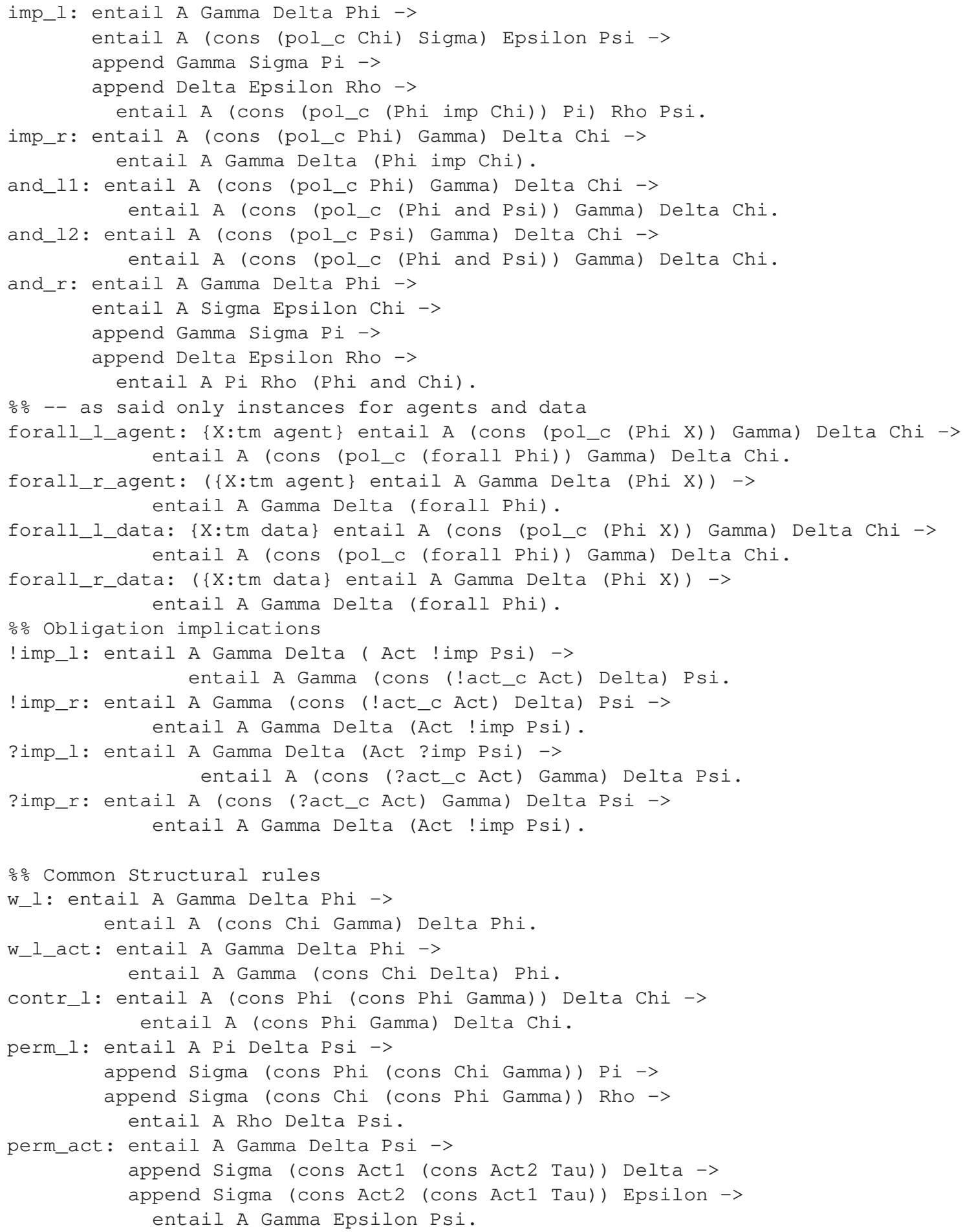




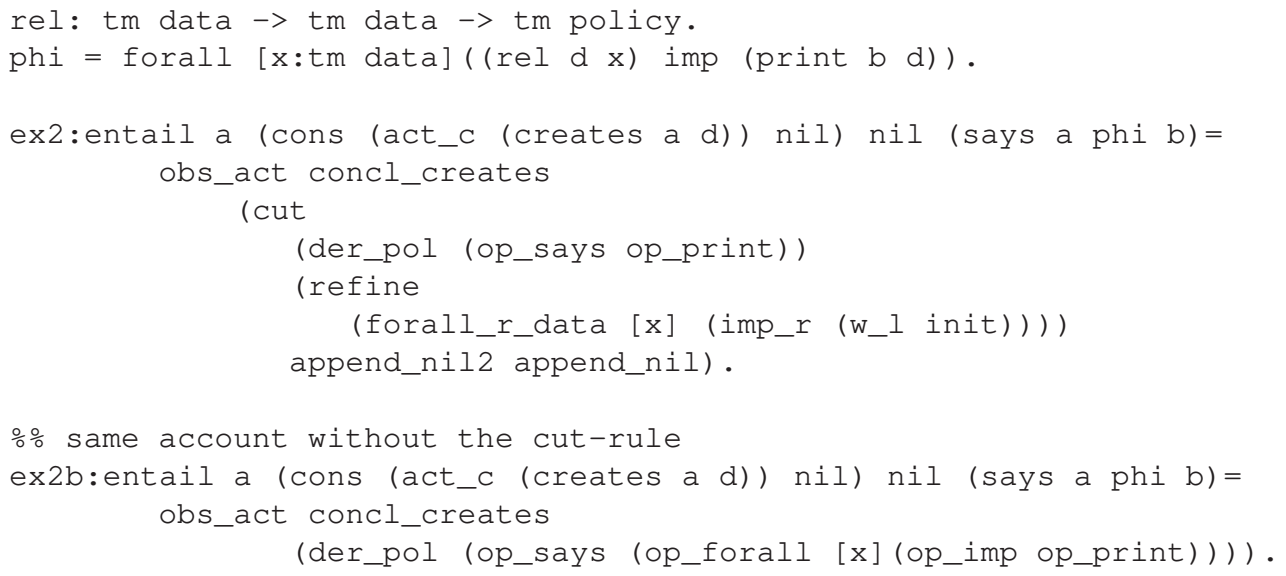

